\title{
Multiple death pathways in TNF-treated fibroblasts: RIP3- and RIP1-dependent and independent routes
}

Cell Research (2011) 21: 368-371. doi:10.1038/cr.2011.3; published online 4 January 2011

\section{Dear Editor,}

Tumor necrosis factor- $\alpha$ (TNF) can induce either apoptosis or programmed necrosis (necroptosis) when different cell lines are used $[1,2]$. While receptorinteracting protein 1 (RIP1) can participate in signaling for both apoptosis and necrosis [3, 4], recent studies by us and by others have shown that RIP3 is essential for TNF-induced necroptosis [5-7] and has no role in TNFinduced apoptosis $[6,7]$. Classification of TNF-induced cell death in a number of cell lines, such as L929 and murine embryonic fibroblasts (MEFs), has been made based on morphological and/or biochemical parameters $[2,7-10]$. It is noteworthy that some apoptotic features, such as testing positive for Annexin V, can also be found in cells that show features of necrosis [11], and, therefore, only major parameters such as caspase dependence can be used to distinguish apoptosis from necrosis. The pan-caspase inhibitor benzyloxycarbonyl-Val-Ala-Aspfluoromethylketone (zVAD) has been used to determine the type of cell death induced by TNF (e.g., apoptosis can be inhibited by inhibition of caspases but necroptosis cannot) $[4,12,13]$. In response to TNF treatment, the fibroblast cell lines - the N line of NIH3T3, the A line of NIH3T3, L929 and MEF cells - showed differences in the caspase dependence of their cell death [2, 7-10]. To obtain an overall picture of the pathways that mediate TNF-induced cell death signaling, we need to know the caspase dependence of the cell death occurring in these cell lines under different conditions, and the dependence of RIP1 and/or RIP3 in these cell death pathways.

We showed in our previous study that TNF-induced cell death in the N line of NIH3T3 and L929 cells is RIP3-dependent necroptosis (Zhang et al. [7] and Supplementary information, Figure S1A-S1D). Since zVAD can enhance necrosis in some cells and zVAD's effect on the cell is known to be beyond caspase inhibition [14], we included another pan-caspase inhibitor quinoline-ValAsp-difluorophenoxymethylketone (QVD) in our experiments. QVD did not inhibit TNF-induced cell death in the N line of NIH3T3 and L929 cells (Supplementary information, Figure S1B and S1E), confirming that TNFinduced cell death in the N line of NIH3T3 and L929 cells was caspase independent. Primary MEF cells are less sensitive to TNF-induced cell death in comparison with fibroblast cell lines such as L929, but high doses of TNF $(150 \mathrm{ng} / \mathrm{ml})$ can still induce RIP3-dependent necroptosis in MEFs (Supplementary information, Figure S1F and S1G). It is generally believed that TNF stimulation activates not only the death pathway but also the survival pathway. Inhibition of new protein synthesis by cycloheximide (CHX) can block the survival pathway and, thus, is widely used in studying cell death. Indeed, treatment of primary MEF cells with $30 \mathrm{ng} / \mathrm{ml}$ TNF did not cause cell death, but induced significant cell death in the presence of $\mathrm{CHX}$ (Figure 1A). TNF+CHX-induced $\mathrm{MEF}$ cell death is caspase independent, since zVAD or QVD cannot inhibit it (Figure 1A). Both zVAD and QVD can partially inhibit $\mathrm{TNF}+\mathrm{CHX}$-induced cell death in $R I P 3^{-/}$MEFs (Figure 1A and 1B). Similar results were obtained when immortalized MEFs were used (Supplementary information, Figure $\mathrm{S} 1 \mathrm{H}$ ). Because part of RIP3-independent cell death in TNF+CHX-treated MEF cells is insensitive to zVAD or QVD inhibition, this portion of cell death should be non-apoptotic and be RIP3independent necroptosis.

RIP1 is known to play a crucial role in TNF-induced apoptosis [3]. TNF treatment in the A line of NIH3T3 cells induces caspase-dependent apoptosis, and expression of RIP1 causes caspase-dependent cell death in the A line of NIH3T3 cells (Supplementary information, Figures S1A, S1B and S2A), supporting the role of RIP1 in apoptosis. However, there must also be a RIP1-independent apoptotic pathway, since TNF $+\mathrm{CHX}$ can induce RIP1-independent apoptosis in an immortalized $R I P 1^{-1-}$ cell line (named as MEF-ZG line in our figures; Lin et al. [4] and Supplementary information, Figure S2B and $\mathrm{S} 2 \mathrm{C})$. For an unknown reason, both RIP1 and RIP3 were undetectable in this cell line. This cell line is abnormal in comparison with other immortalized MEF lines, in that it is $\sim 100$-fold more sensitive to TNF+CHX-induced cell death, although it cannot be killed by TNF alone. Never- 
A

A

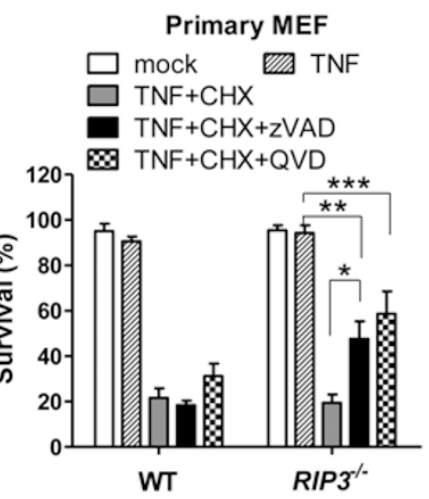

D

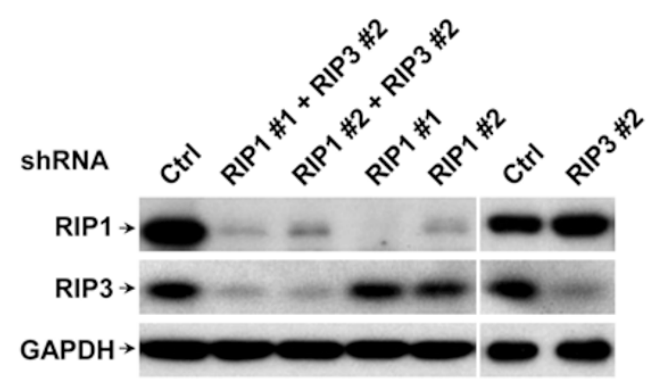

$\mathbf{F}$

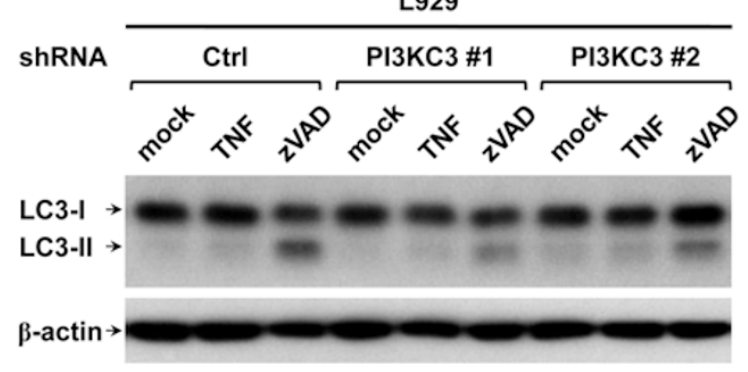

B

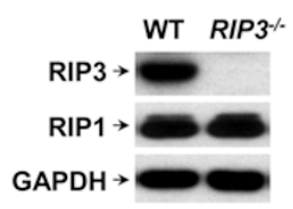

C

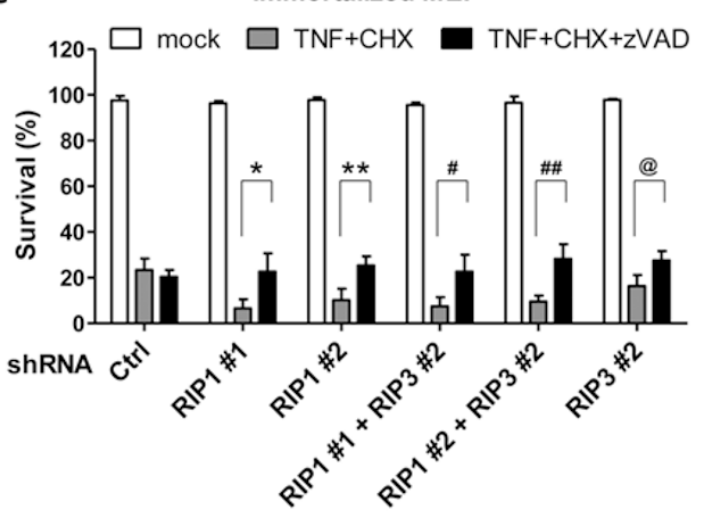

E

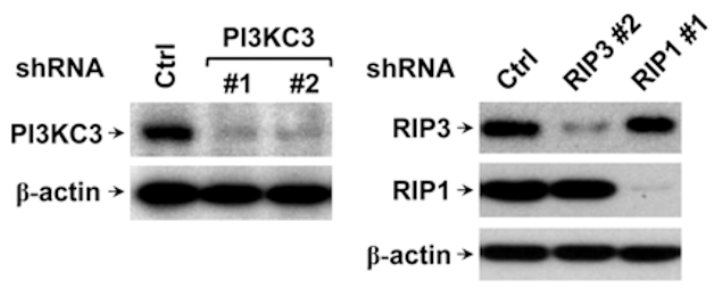

G

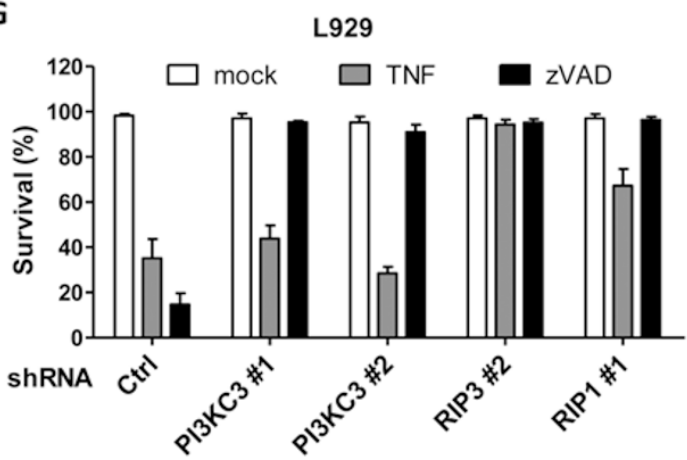

Figure 1 Multiple cell death pathways in TNF-treated fibroblasts. (A) CHX revives RIP3-independent necroptosis in TNF-

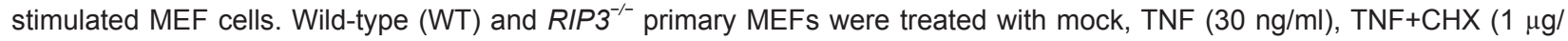
$\mathrm{ml}), \mathrm{TNF}+\mathrm{CHX}+\mathrm{ZVAD}(20 \mu \mathrm{M})$ or TNF+CHX+QVD $(20 \mu \mathrm{M})$ for $24 \mathrm{~h}$. Cell viabilities were then determined. ${ }^{*} P=0.005,{ }^{*} P$ $=0.001$ and ${ }^{* * *} P=0.004$ (Supplementary information, Data S1). Data were presented as mean \pm SEM of triplicates. (B) Western blot analysis of RIP3 and RIP1 expression levels in WT and RIP3 ${ }^{-/}$MEFs (Supplementary information, Data S1). GAPDH was used as a loading control. (C) TNF+CHX-induced death in RIP1 knockdown MEF cells is partially sensitive to zVAD. Wild-type MEF cells were infected with lentivirus encoding control or indicated shRNA(s) for $48 \mathrm{~h}$ (Supplementary information, Data S1). Viabilities of these cells that stimulated with mock, TNF $(30 \mathrm{ng} / \mathrm{ml})+\mathrm{CHX}(1 \mu \mathrm{g} / \mathrm{ml})$ and TNF $+\mathrm{CHX}+\mathrm{ZVAD}$ $(20 \mu \mathrm{M})$ for $24 \mathrm{~h}$ were measured. ${ }^{*} P=0.036,{ }^{*} P=0.015,{ }^{\#} P=0.035,{ }^{\#} P=0.009$ and ${ }^{\circledR} P=0.041$. Data were presented as mean \pm SEM of triplicates. (D) RIP1, RIP3 and GAPDH expression levels in the cells used in panel C were analyzed by western blotting. (E) L929 cells were infected with lentivirus encoding control, shRNAs of PI3KC3, RIP3 or RIP1 for 48 h. Protein levels were evaluated by western blotting. $\beta$-actin was used to confirm equal loading. (F) Depletion of PI3KC3 inhibited zVADinduced modification of LC3. Control and PI3KC3 knockdown L929 cells used in panel E were treated with mock, TNF (1 ng/ $\mathrm{ml})$ or ZVAD $(20 \mu \mathrm{M})$ for $12 \mathrm{~h}$. Cell lysates were subjected to western blot analysis with antibodies against LC3 and $\beta$-actin. (G) Depletion of PI3KC3 in L929 cells had no effect on TNF-induced necroptosis, but effectively blocked zVAD-induced cell death. L929 cells described in panel $\mathbf{E}$ were treated for $24 \mathrm{~h}$ with or without TNF $(10 \mathrm{ng} / \mathrm{ml})$ or ZVAD $(20 \mu \mathrm{M})$. Cell viabilities were then measured. Data were presented as mean \pm SEM of triplicates. All experiments were done at least three times. 
Unlike this immortalized $R I P 1^{-/-}$cell line, only a small portion of TNF $+\mathrm{CHX}$-induced cell death in RIP1 knockdown MEF cells was inhibited by zVAD (Figure $1 \mathrm{C}$ and 1D). Although we cannot exclude the possibility that the trace amount of remaining RIP1 still mediated necroptosis in the RIP1 knockdown MEFs, it is most likely that a portion of the zVAD-insensitive cell death is mediated by a RIP1-independent mechanism. We also knocked down RIP3 or RIP1 and RIP3 simultaneously, and observed a similar portion of caspase-independent cell death (Figure 1C and 1D), suggesting that this portion of TNF+CHX-induced cell death is RIP1 and RIP3 independent.

It was observed that $\mathrm{zVAD}$ can induce autophagic cell death in L929 cells [15], and autophagic cell death was associated with RIP1- and RIP3-dependent cell death $[12,13]$. Since autophagic cell death is known to be independent from caspases, we sought to address whether TNF-induced necroptosis is an autophagic cell death or whether autophagy is required for TNF-induced necroptosis. Phosphoinositide 3-kinases (PI3Ks) are a family of intracellular signal transducer enzymes that catalyze phosphorylation of the 3'-hydroxyl position of the inositol ring of phosphatidylinositol. Activation of class III PI3K is essential for autophagosome formation. 3-methyladenine (3-MA) and wortmannin (WM) both can inhibit PI3K activity; thus, they are often used to inhibit autophagy. We tested the effect of 3-MA and WM on TNF-induced necroptosis, and found that 3-MA and WM had no effect on the TNF-induced death of L929 cells (Supplementary information, Figure S3A and S3B). In mammalian cells, signals that lead to inactivation of mammalian target of rapamycin (mTOR) can induce autophagy. Our experimental data showed that inhibition of mTOR by rapamycin in L929 cells had no effect on TNF-induced cell death (Supplementary information, Figure S3C).

Since chemical inhibitors could target other proteins inside cells, we employed RNA interference (RNAi) knockdown to determine the requirement of autophagy in TNF-induced L929 cell death. PI3KC3 (PI3K, class 3 ), also named VPS34 (vacuolar protein sorting 34), is the sole member of the class III PI3K. We designed two shRNAs that can efficiently deplete the protein level of PI3KC3 in L929 cells (Figure 1E, left panel). zVADinduced modification of microtubule-associated protein 1 light chain 3 (LC3) in L929 cells was inhibited in PI3KC3 knockdown cells (Figure 1F), indicating an inhibition of autophagy. We found that knockdown of PI3KC3 in L929 cells had no effect on TNF-induced necroptosis (Figure 1G), while depletion of RIP3 or RIP1 by RNAi inhibited TNF-induced cell death (Fig- ure $1 \mathrm{E}$, right panel, and $1 \mathrm{G})$. In contrast, knockdown of PI3KC3, RIP3 or RIP1 all blocked zVAD-induced cell death (Figure $1 \mathrm{G}$ ), which is known to be an autophagic cell death [15]. Based on the inhibitor and RNAi studies, we conclude that TNF-induced necroptotic cell death does not require PI3KC3-dependent autophagy.

Although the traditional classification of cell death is very useful in understanding and studying different types of cell death, it is clear now that both apoptosis and necrosis are executed by more than a single mechanism. In addition to RIP1-dependent apoptosis and RIP3-dependent necroptosis [3, 5-7], RIP1-independent apoptosis and RIP3-independent necroptosis can also be observed. Although RIP1 and RIP3 can participate in autophagic cell death, autophagy is not required for TNF-induced RIP1/3-dependent necroptosis. Multiple signaling pathways can be used by TNF to induce apoptosis or necroptosis, and the predominant death pathway in different cell lines can be significantly different.

\section{Acknowledgments}

We thank Dr K Newton and Dr VM Dixit (Genentech, Inc., USA) for kindly providing RIP3 knockout mice, and Dr ZG Liu (National Cancer Institute, NIH, USA) for the $R I P 1^{-/-} \mathrm{MEF}$ cells (MEF-ZG line). This work was supported by 973 program 2009CB522200, 111 Project (B06016), NSFC Grant 30830092 and CPSF Grant 20100470040.

Duan-Wu Zhang ${ }^{1, *}$, Min Zheng ${ }^{1, *}$, Jing Zhao ${ }^{1}$, Yuan-Yue $\mathrm{Li}^{1}$, Zhe Huang ${ }^{1}$, Zhu Li ${ }^{1}$, Jiahuai Han ${ }^{1}$

${ }^{I}$ The Key Laboratory of the Ministry of Education for Cell Biology and Tumor Cell Engineering, School of Life Sciences, Xiamen University, Xiamen 361005, China

*These two authors contributed equally to this work. Correspondence: Jiahuai Han

Tel: +86-592-2187680; Fax: +86-592-2187930

E-mail: jhan@xmu.edu.cn; jhan@scripps.edu

\section{References}

1 Kroemer G, Dallaporta B, Resche-Rigon M. The mitochondrial death/life regulator in apoptosis and necrosis. Annu Rev Physiol 1998; 60:619-642.

2 Fiers W, Beyaert R, Declercq W, Vandenabeele P. More than one way to die: apoptosis, necrosis and reactive oxygen damage. Oncogene 1999; 18:7719-7730.

3 Festjens N, Vanden Berghe T, Cornelis S, Vandenabeele P. RIP1, a kinase on the crossroads of a cell's decision to live or die. Cell Death Differ 2007; 14:400-410.

4 Lin Y, Choksi S, Shen HM, et al. Tumor necrosis factorinduced nonapoptotic cell death requires receptor-interacting protein-mediated cellular reactive oxygen species accumulation. J Biol Chem 2004; 279:10822-10828. 
5 Cho YS, Challa S, Moquin D, et al. Phosphorylation-driven assembly of the RIP1-RIP3 complex regulates programmed necrosis and virus-induced inflammation. Cell 2009; 137:11121123.

6 He S, Wang L, Miao L, et al. Receptor interacting protein kinase-3 determines cellular necrotic response to TNF-alpha. Cell 2009; 137:1100-1111.

7 Zhang DW, Shao J, Lin J, et al. RIP3, an energy metabolism regulator that switches TNF-induced cell death from apoptosis to necrosis. Science 2009; 325:332-336.

8 Grooten J, Goossens V, Vanhaesebroeck B, Fiers W. Cell membrane permeabilization and cellular collapse, followed by loss of dehydrogenase activity: early events in tumour necrosis factor-induced cytotoxicity. Cytokine 1993; 5:546-555.

9 Sakon S, Xue X, Takekawa M, et al. NF-kappaB inhibits TNFinduced accumulation of ROS that mediate prolonged MAPK activation and necrotic cell death. EMBO J 2003; 22:38983909.

10 Ventura JJ, Cogswell P, Flavell RA, Baldwin AS Jr, Davis RJ.
JNK potentiates TNF-stimulated necrosis by increasing the production of cytotoxic reactive oxygen species. Genes Dev 2004; 18:2905-2915.

11 Wang X, Ono K, Kim SO, et al. Metaxin is required for tumor necrosis factor-induced cell death. EMBO Rep 2001; 2:628633.

12 Degterev A, Huang Z, Boyce M, et al. Chemical inhibitor of nonapoptotic cell death with therapeutic potential for ischemic brain injury. Nat Chem Biol 2005; 1:112-119.

13 Hitomi J, Christofferson DE, Ng A, et al. Identification of a molecular signaling network that regulates a cellular necrotic cell death pathway. Cell 2008; 135:1311-1323.

14 Temkin V, Huang Q, Liu H, Osada H, Pope RM. Inhibition of ADP/ATP exchange in receptor-interacting protein-mediated necrosis. Mol Cell Biol 2006; 26:2215-2225.

$15 \mathrm{Yu} \mathrm{L}$, Alva A, Su H, et al. Regulation of an ATG7-beclin 1 program of autophagic cell death by caspase-8. Science 2004; 304:1500-1502.

(Supplementary information is linked to the online version of the paper on the Cell Research website.) 